\title{
Predictive factors of surgical outcome in frontal lobe epilepsy explored
}

\section{with stereo-electroencephalography}

4

(1)

(1)

\section{ABSTRACT}

Background: Resective surgery is a well-established treatment for pharmacoresistant frontal lobe epilepsy (FLE), but seizure outcome and prognostic indicators are poorly characterised and vary between studies.

Objective: To study long-term seizure outcome and identify prognostic factors.

Methods: We retrospectively analysed 42 FLE patients having undergone surgical resection, mostly preceded by invasive recordings with stereo-electroencephalography (SEEG). Post-surgical outcome up to 10 years' follow-up and prognostic indicators were analysed using Kaplan-Meier analysis, multivariate and conditional inference procedures.

Results: At the time of last follow-up $57.1 \%$ of patients were seizure-free. The estimated chance of seizure freedom was 67\% (95\% CI 54-83) at 6 months, 59\% (95\% CI 46-76) at 1 year, 53\% (95\% CI $40-71)$ at 2 years and $46 \%$ (95\% CI 32-66) at 5 years. Most relapses (83\%) occurred within the first 12 months. Multivariate analysis showed that completeness of resection of the epileptogenic zone (EZ) as defined by SEEG was the main predictor of seizure outcome. According to conditional inference trees, in patients with complete resection of the epileptogenic zone, focal cortical dysplasia as aetiology and focal EZ were positive prognostic indicators. No difference in outcome was found in patients with positive versus negative MRI.

Conclusion: Surgical resection in drug-resistant FLE can be a successful therapeutic approach, even in the absence of neuroradiologically visible lesions. SEEG may be highly useful in both non-lesional and lesional FLE cases, since complete resection of the EZ as defined by SEEG is associated with better prognosis.

Running title: Outcome in frontal lobe epilepsy surgery

Keywords: Frontal lobe epilepsy; epilepsy surgery; outcome; Stereo-electroencephalography; MRInegative; Focal Cortical Dysplasia 
INTRODUCTION

Surgical resection of the epileptogenic tissue is a well-established treatment for pharmacoresistant patients ${ }^{1,2}$. Frontal lobe epilepsy (FLE) accounts for 6 to $30 \%$ of all epilepsy surgery and represents the second most common partial epilepsy after temporal lobe epilepsy (TLE) ${ }^{3-7}$. However, surgical outcome is considered less favourable and long-term success rates are more variable. Favourable seizure outcome ranges from 20.0 to $77.8 \%$ depending on series, with the majority of more recent studies reporting seizure freedom rates of around $50 \%^{5,8-12}$. The causes of post-operative seizure recurrence are poorly characterised, as are potential prognostic indicators. Assessing the probability of seizure freedom and determining prognostic factors is crucial to select candidates for epilepsy surgery and to drive the complex decision-making process from pre-surgical evaluation to definition of cortectomy.

Here we studied long-term seizure outcome in a cohort of FLE patients having undergone surgical resection, preceded in most cases by intracerebral recording with stereoelectroencephalography (SEEG). We investigated potential indicators of outcome using univariate and multivariate statistical methods together with conditional inference procedures, which allow evaluation of seizure recurrence over time, stability of predictors and correlations and interactions among data.

\section{METHODS}

\section{Patient selection}

We reviewed patients evaluated in the Epilepsy Unit, XXXXXX Hospital, XXXXXX, France, with a diagnosis of drug-resistant frontal lobe epilepsy, who underwent resective FLE surgery from 2000 to 2013. Most patients underwent pre-surgical evaluation with intracerebral EEG. Exclusion criteria were hemispherotomy, callosotomy, radiosurgery and follow-up duration $\leq 6$ months. Collected clinical, radiological, electrophysiological and histopathological data are presented in table 1.

\section{Pre-operative protocol}

Patients underwent non-invasive pre-surgical assessment including prolonged scalp video-EEG monitoring (International 10-20 system). Interictal and ictal EEG abnormalities were classified into 3 categories: lateralised frontal, lateralised hemispheric or bilateral. MRI in patients up until 2010 was performed with a 1.5 T machine; in 14 patients explored from 2010 onwards, MRI was performed with a 3 T Siemens Magnetom scanner (Siemens AG, Erlangen, Germany). MRI epilepsy protocol included transverse diffusion images, transverse T2-weighted images, coronal T1-weighted inversion recovery images, coronal fluid-attenuated inversion recovery (FLAIR) images and a three-dimensional T1-weighted acquisition. Acquisition plans were referred to the bi-hippocampal plane for the transverse acquisitions and to the $\mathrm{AC}-\mathrm{PC}$ plane for the coronal and axial acquisition. Reconstructions of the 3D T1 images were obtained as well. The multi-channel head coil allowed the use of matrix acquisition, isotropic 1mm 3D T1 images, with reasonable acquisition time especially for inversion- 
recovery, FLAIR and 3D sequences. MRI findings were classified as normal or pathological and, when pathological, as showing a lesion limited to the frontal lobe, or a lesion extending beyond the frontal lobe. Positron emission tomography (PET) and interictal single photon emission computer tomography (SPECT) were obtained in all but 12 patients. If results of non-invasive investigations did not lead to the formulation of a single hypothesis about the localization of the epileptogenic zone (EZ) or if they pointed close to functional cortex, patients were selected for SEEG. SEEG exploration was carried out during long-term video-EEG monitoring and recordings were performed using intracerebral multiple contact electrodes (10-15 contacts, length: $2 \mathrm{~mm}$, diameter: $0.8 \mathrm{~mm}, 1.5 \mathrm{~mm}$ apart) placed intracerebrally according to Talairach's stereotactic method ${ }^{8}$. Number of electrodes per patients varied from a minimum of 5 to a maximum of 13 electrodes in present series (mean 9,3 \pm 2 ). Electrodes were implanted bilaterally in all but nine patients. All patients gave their informed consent prior to exploration and the study was approved by the Institutional Review Board of the French Institute of Health. Based on SEEG seizure recordings the EZ was defined as the regions involved in primary organization of the seizure, classified into focal (the seizure onset being limited to one anatomical area, lesional or not) or widespread (the seizure onset was not limited to a single functional region $)^{9}$. This definition was based on visual analysis $+/$ - a quantitative measure of epileptogenicity, the Epileptogenicity Index (EI) ${ }^{10}$ implemented on Coherence Natus/Deltamed software. Neuropsychological test data before and after surgery were available for 25/42 patients. All these patients were administered a comprehensive battery of standardized neuropsychological tests, exploring intelligence and memory (immediate and delayed verbal and visual memory). The standard clinical measures of intelligence retained for IQ evaluation was Wechsler Intelligence Scale; according to the age, Wechsler intelligence scale for children - fourth edition (WISC IV) 6 to 16 years and Wechsler Adult Intelligence Scale third edition (WAIS-III) from 16 years (Wechsler, D. 2005; Wechsler, D. 2000). Memory abilities with verbal and/or visual material and attention/working memory were assessed with the score of the Wechsler Memory Scale (MEM III from 16 years, CMS 6 to 16 years) (Wechsler, D. 2001; Wechsler, D. 2006).

\section{Surgery and post-operative protocol}

All patients underwent unilateral frontal lobe surgery, including lesionectomy, subtotal frontal lobectomy or frontal lobectomy. Resection area was classified based on anatomical landmarks into premotor, prefrontal, premotor + prefrontal and fronto-temporal (when extended to the anterior temporal lobe). Tissue specimens were analysed for histopathological diagnosis. Following surgery, patients were managed in the neurosurgery or intensive care unit and the occurrence of acute postoperative seizures (APOS) was noted. Surgical resection of the EZ was classified into complete or incomplete based on SEEG criteria allowing the identification of the EZ: a complete resection was defined as the total removal of the cortical tissue covered by intracerebral electrodes of the EZ, while 
resection was considered incomplete if not all cortical tissue belonging to the EZ was removed.

Additionally, in lesional cases, definition of complete resection also required the removal of the entire lesion, based on pathological data and, in cases with preoperative MRI lesion, on postoperative MRI. Possible causes of incomplete resection of the EZ included: a limited resection in order to preserve functional cortex (namely the primary motor cortex and Broca's area) and minimize postsurgical deficits; the involvement of contralateral cortex; remote epileptogenicity extending beyond the frontal lobe or the anterior temporal lobe; from histopathological analysis, the presence of residual pathological tissue within the border of the removed cortical tissue and/or of residual lesion visible on MRI.

\section{Outcome definition and follow-up}

Seizure related-outcome was assessed based on 6 months then yearly (or as indicated by clinician) follow-up. Patients were classified as being either seizure-free (as defined by Engel class IA) or notseizure-free ${ }^{11}$. The timing of the first postoperative seizure (beyond the first postoperative week for patients with APOS) was considered the time of recurrence and was set down for use in Kaplan-Meier analysis.

\section{Statistical analysis}

Univariate and multivariate statistics were used to test for predictors of outcome.

Descriptive statistics were obtained for each variable. As initial analysis, we performed Wilcoxon rank sum, $\chi^{2}$ and Fisher's exact test to compare seizure-free to not seizure-free at 6 months up to 10 years after surgery. Individual patient differences between pre-surgical and post-surgical scores of neuropsychological tests were calculated. These were then analysed at group level using the Wilcoxon rank sum test, comparing the seizure-free and the non seizure-free groups. Variables with a significant level at 5\% on univariate analysis entered in a Multiple Correspondence Analysis (MCA). The MCA method projects multi-dimensional data - one dimension for each variable - into a bi-dimensional space (or more) and searches for patterns in the datasets ${ }^{12}$. The matrix of eigenvalues is determined to identify a combination of variables that present more stability in the factorial plan and explained the largest percentage of variability in the dataset. This allows identification of variable modalities that are more closely associated with different populations (i.e. with seizure-free patients). The MCA provides a visual representation regarding the conditions more strongly coupled with groups that helps to confirm associations or similarities between variable modalities. This method is suitable for population-based studies ${ }^{13}$. Variables with a significant level at $10 \%$ on univariate analysis were entered into a survival multiple regression analysis, the Andersen-Gill counting process model, in order to identify predictors of outcome $^{14}$. Statistical significance was set at the $5 \%$ level. This method aims to simultaneously 
explore the effects of several variables on possibly recurrent outcome, while taking into account the relationship and possible dependency between the values of two or more variables.

Finally conditional inference trees analysis was performed in order to identify subpopulations of patients susceptible to present seizure recurrence. Conditional inference trees are a supervised classification method for analyzing data that select covariates by permutation-based significance tests, thereby avoiding potential bias of the more traditional decision tree algorithms ${ }^{15}$. The results are displayed in a 'tree' graph, showing the hierarchy of significant variables, and the final groups and associated thresholds of response values following the binary splits.

All statistics were performed using R software (version 2.13.1; R Core Development Team, 2013).

\section{RESULTS}

\section{Patient characteristics}

Within 54 patients with FLE who underwent epilepsy surgery, 42 patients ( 28 females) fulfilled inclusion criteria and were included in the study. Mean age at epilepsy onset was 7 years ( \pm 6 years, median 5$)$, mean age at surgery was 23.4 years $( \pm 12.3$ years, median 23$)$ and the mean epilepsy duration at the time of surgery was 16.4 years $( \pm 10.7$ years, median 15$)$. Thirty-eight patients $(90 \%)$ underwent SEEG prior to surgery and only four patients underwent surgical resection without previous SEEG (two because of young age, two because of patient refusal of invasive recording, all with lesional MRI).

MRI was normal in 18 patients (43\%). Patients having undergone 3T MRI did not have more positive findings compared to patients having undergone 1.5T MRI. Lesional cases on MRI presented with a lesion limited to the frontal lobe (13 patients, $31 \%$ ) or a lesion with extra-frontal extension (11 patients, 26\%).

Histopathology was available in all but five patients, who all had lesional MRI.

Aetiology, determined from combined histopathological and MRI findings, was focal cortical dysplasia in 25 patients (59.5\%, 21 with FCD IIb and 4 with FCD Ib) (11 of whom with normal MRI), cryptogenic in 7 patients (16.7\%) with normal MRI and normal or with mild gliosis on histopathology, and other pathological findings in 10 patients (24\%) including encephalitis in 2, tuberous sclerosis in 2 , encephalomalacia from trauma or stroke in 3 , vascular malformation in 2 , and other malformation of cortical development in 1. Clinical patient characteristics are summarised in table 1.

\section{Overall recurrence}

Mean follow-up duration was 4.6 years ( \pm 2.7 years, median 4.5 years, range $0.5-10$ years). At the time of last follow-up 24 patients were seizure-free (57.1\%) while in 18 patients seizures recurred without subsequent remission (42.9\%). Within the seizure-free group, a run-down phenomenon was observed in two patients (who became seizure-free at 1 and 1.5 years for a subsequent follow-up period of respectively 10 and 10.5 years) and in another patient, seizures recurred with abrupt discontinuation of AED, and ceased after resuming AED intake. Nonetheless, in order to evaluate 
longitudinal outcome changes and explore the effects of variables on outcome upon a time, their seizure recurrence was taken into account for statistical analysis.

Seizure outcomes using Engel criteria at 6 months and at 1 to 5 years post-operatively are reported in table 2 . The majority of relapses (17, when including patients with a run-down) occurred during the first year. Of the four patients without SEEG, two were seizure free and two were not-seizure free at the end of their participation (respectively $0.5,2.5,2$ and 1.5 years). Longitudinal seizure-free outcome estimated using Kaplan-Meier survival analysis reveals that probability of remaining in Engel Class I vary with post-surgical time (Figure 1). The chance of being seizure free was $67 \%$ (95\% CI 54-83) at 6 months, then fell to $59 \%$ (95\% CI 46-76) at 1 year, 53\% (95\% CI 40-71) at 2 years, 50 (95\% CI 36-68) at 3 years and $46 \%$ (95\% CI 32-66) at 5 years and beyond. The median time to seizure recurrence was 36 months.

\section{Neuropsychological outcome}

Concerning neuropsychological outcome, we found that patients assigned an Engel class I outcome compared to not-seizure free patients had a post-surgical higher full scale IQ $(\mathrm{P}=0.029)$ as well as a higher verbal IQ $(\mathrm{P}=0.017)$. Conversely, there was no significant difference comparing pre-surgical to post-surgical scores between the groups on Performance IQ and for working memory scores.

\section{Outcome predictors}

\section{Univariate analysis}

Results of univariate analysis for categorical variables are shown in table 1-3. For statistical purposes, MRI findings were twice grouped into two modalities to compare: normal versus lesional MRI and then MRI with extra-frontal extension MRI versus MRI (either normal or lesional) without extrafrontal extension. Similarly, aetiology was doubly grouped into FCD, cryptogenic, and other, and then into FCD versus not-FCD.

Variables significantly $(\mathrm{P}<.05)$ associated with seizure recurrence were: incomplete resection of the EZ, cryptogenic and not-FCD as aetiology, extra-frontal extension lesional MRI and widespread EZ as defined by SEEG. Not significant difference in outcome was found in MRI-lesional patients compared to MRI-normal patients. When categorizing surgical outcome based on field strength, we found no difference for patients having undergone 3T MRI compared to 1.5T MRI. Combining aetiology with MRI findings, the poorest outcomes were associated with normal or non-specific histopathological findings associated with normal MRI on one hand, and aetiology other than FCD associated with extra-frontal extension MRI on the other hand.

Concerning continuous variables (namely, age at epilepsy onset, age at surgery and epilepsy duration), none of them proved to be correlated to surgical outcome $(\mathrm{P}>0.1)$. 
Multiple Correspondence Analysis

Variables with $\mathrm{P}<.05$ on univariate analysis entered in the MCA. For a principle of parsimony, FCD

versus not-FCD was chosen to three modalities aetiology because of its lower $p$ value, and the combined variable aetiology/MRI was not included in the MCA.

As a result, the two first dimensions in the factorial plan explained $61.7 \%$ of the variance. This indicated that such an obtained two-dimensional plan furnishes a reliable representation of the fivedimensional reality, where five is the number of considered variables. Variables proximity to the seizure free group or to the not-seizure free group indicates the characteristics that each group is more likely to present (Figure 2). The factorial plan illustrates the characteristics associated to each other and contributing the most to describing the group not-seizure free: incomplete resection of the EZ, notFCD as aetiology, widespread EZ, lesional MRI with extra-frontal extension, presence of GTCS. Conversely, variable modalities associated with the seizure-free group were complete resection of the EZ, FCD as aetiology, focal EZ, not extra-frontal extension MRI, absence of GTCS.

\section{Multivariate analysis}

Results of multivariate analysis after applying Andersen-Gill counting process model are reported in table 4. The variable "extent of resection" resulted in an independent predictor of outcome $(\mathrm{P}=.04)$ in our series, with a hazard ratio equal to 4 for incomplete resection $\left(\beta=1.39, \mathrm{p}<0.05, \mathrm{e}^{\beta}=3.9998\right.$, CI $95 \%[1.097,14.582])$, meaning that incomplete resection of the EZ as defined by SEEG led to a risk of seizure recurrence four times greater than for complete resection.

The Kaplan-Meier survival curve for this outcome predictor shows a less favourable outcome for patients with incomplete resection of the EZ $(\mathrm{P}<.001)$, with $65 \%$ of patients recurring within the first six months post-operatively (CI 18-72) (Figure 3).

\section{Conditional inference trees}

Recursive partitioning (Figure 4) confirmed extent of resection as the most important variable associated with outcome (first node). Secondly, aetiology was relevant in patients with complete resection (left branch of the tree), with not-FCD as aetiology associated with seizure recurrence. Thirdly, in the FCD branch, focal EZ predicted seizure freedom while widespread EZ predicted seizure recurrence.

\section{DISCUSSION}

In the present study we aimed to investigate post-surgical outcome in FLE and to identify potential predictors of seizure recurrence using different statistical techniques, combining the advantages of 1) univariate analysis to select the most relevant variables, 2) MCA to highlight the structure of the 
population with respect to prognosis, 3) survival multiple regression analysis to derive significant parts of this structure and 4) conditional inference trees to refine this conclusion.

As a result $57.1 \%$ of patients were seizure freedom at last follow-up and the estimated chance of longterm (10 years) favourable outcome was $46 \%$. Most relapses occurred within the first two postoperative years. Afterwards, likelihood of maintaining a good outcome following a prolonged period of seizure freedom was quite high.

MCA distinguished a stable combination of variables associated with favourable outcome, which could help to identify optimal candidates for surgery: MRI normal, or showing a lesion limited to the frontal lobe; focal EZ; complete resection of the EZ; FCD as aetiology; no GTCS.

Multivariate and Conditional Inference procedures showed that the complete resection of the EZ as defined by SEEG was the main predictor of favourable outcome. Additionally FCD predicted seizure freedom in patients with complete resection, and a focal EZ was associated with favourable outcome in patients with FCD. Importantly, patients with negative MRI had the same chance of favourable outcome as patients with lesional MRI in our series.

Overall, present success rates are comparable or even slightly better than those reported in previous studies and in a recent meta-analysis ${ }^{5}$, indicating that a long-term success rate close to that of TLE ${ }^{16}$ can be reached in FLE surgery investigated by SEEG. Indeed TLE surgery has been recently reported to have long term (5-15 years) seizure freedom varying from 37 to $63 \%$, often depending on pathology ${ }^{16-18}$.

Our study shows a deterioration of outcome with time, as also observed after TLE surgery ${ }^{17,18}$. Reports on FLE surgery exhibit a great variability across studies, concerning both outcome rates and prognostic indicators ${ }^{5}$. This could be due to different statistical approaches, evolution of neuroimaging techniques, selection bias or, additionally to the diverse and rather imprecise evaluation of some variables, namely the extension of the resection of the epileptogenic tissue. Indeed the complete resection of the neuroimaging abnormality has been associated with higher success rate in several studies ${ }^{19-23}$. Consistently, the absence of a visible lesion on MRI is often reported as a negative predictor of outcome ${ }^{5,20,24-28}$. Here the completeness of the resection was defined based upon SEEG criteria, and the total removal of the so defined epileptogenic tissue was by far the main predictor of seizure outcome, confirming that it represents the sine qua non condition to attain seizure freedom ${ }^{8}$. Moreover, we found no difference in outcome when comparing patients with normal MRI to patients with lesional MRI. This is in agreement with a previous SEEG study ${ }^{29}$ but is in contrast with other series ${ }^{20,28}$ and indicates that SEEG is equally effective in MRI negative and positive cases 29 .

Compared to our population predominantly explored with SEEG, the great majority of series studying long-term seizure outcome of FLE are characterised by the absence of invasive recording or by the use of subdural electrodes in about half of patients undergoing cortical resection $\left({ }^{4,20,24-27,30-34}\right)$. In these subdural guided series, the rate of negative MRI varies from $0 \%$ to $46 \%$ (39\% on average ${ }^{4,20,24-26,30-}$ 
$32,34,35$ ). In the majority of epilepsy centres, invasive subdural electrodes have traditionally been the intracranial EEG method of choice including for evaluating non-lesional cases ${ }^{20,24,30-32,35}$. Until very recently the SEEG was almost exclusively used in France and Italy. In the last few years, international interest in SEEG as a potentially useful tool for presurgical evaluation, especially for extra-temporal and MRI-negative cases, has led to a rapid worldwide increase in centres adopting this method. For example, the Cleveland Clinic now prioritizes SEEG as first choice exploration method rather than grids for the majority of cases, and particularly for "suspected frontal lobe epilepsy in non-lesional MRI scenario" "Alomar et al 2016). A recent report from an ILAE working party, evaluating different modalities of invasive EEG recordings, discusses the utility of subdural grid for extensive unilateral exploration with wide coverage of neocortical gyral surface ${ }^{37}$ (Jayakar et al, 2016). On the other hand, authors recommend SEEG for bilateral exploration and for deep targets such as the cingulate cortex, the orbito-frontal cortex, the insular cortex and mesio-temporal structures ${ }^{37,38}$ (Jayakar et al, 2016; Podkoryotova et al, 2016). These latter structures, because of their strong anatomo-functional connections with the prefrontal cortex, are particularly important to explore as potentially implicated in the EZ network in FLE. Although more often used in MRI-negative extratemporal epilepsies, invasive recording has been recommended for both MRI negative or positive cases $^{37}$ (Jayakar et al, 2016). In actual fact, with few exceptions ${ }^{24,30}$ (Lazow et al, 2012; Lee et al, 2008), most FLE surgical series do not use systematically intracranial EEG; however, such series without intracranial EEG are generally composed of cases with radiologically visible lesions. Conversely, we regularly used SEEG if clinically indicated, in both MRI positive and MRI negative cases, and found similar or better outcome than other reports.

The use of intracerebral electrodes allows the definition of the EZ and of surgical resection in the absence of a visible lesion. Furthermore it minimizes the risk of an incomplete removal of the EZ extending beyond a visible neuroradiological abnormality, since epileptogenicity can extend beyond the lesion and organizes as a large network with remote and even bilateral epileptogenicity ${ }^{9,39}$. As described in other studies we report a relatively high incidence of FCD amongst operated patients with normal MRI ( $40 \%$ of patients with FCD $)^{9,21,29,40-42}$. In this context the invisible underlying pathology, namely FCD, represented a favourable prognostic indicator in case of complete removal of the EZ, when compared with all other aetiologies, in agreement with previous SEEG studies ${ }^{29,43}$. Moreover, the characteristics of the EZ (i.e. focal versus widespread) can predict surgical outcome in our series. Indeed bilateral involvement and involvement of remote extra-frontal cortex were important causes of incomplete resection of the EZ. Since another main reason of incomplete resection of the EZ is the need to preserve functional cortex, SEEG allows on one hand to evaluate the involvement of such structures and, on the other hand, to predict surgical failure and guide palliative surgery ${ }^{43}$ if indicated.

This study aimed to provide outcome measures that could guide the complex decision-making process leading to surgical resection. The use of SEEG appears to be particularly effective in evaluating the 
eligibility for epilepsy surgery in FLE patients. Of course its effectiveness depends on conditions of use, such as patient selection, electrode implantation strategy and team expertise, which might help explain variable results across series ${ }^{44}$. Here, the complete resection of the EZ defined with SEEG represents the strongest predictor of seizure freedom, both in negative and in lesional MRI. Of course the capability to totally remove the EZ can be constrained by possible overlap between the EZ and functional cortex. Indeed, extension of the EZ to the central cortex has been shown to have a poor prognostic value for surgical outcome ${ }^{45}$.

\section{CONCLUSION}

A number of clinical characteristics that may co-exist can indicate patients with better chance of favourable outcome in FLE, namely: focal EZ; normal MRI or with lesion limited to the frontal lobe; complete resection of the EZ; and FCD as aetiology. SEEG in FLE candidates for surgery, both in lesional and non-lesional cases, contributes above all to the definition of the EZ, whose complete resection is the major outcome predictors for FLE. Epilepsy surgery can represent a successful therapeutic approach in drug-resistant FLE patients, even in the absence of a neuroradiological lesion.

\section{Disclosure of Conflicts of Interest}

"None of the authors has any conflict of interest to disclose." 


\section{References}

1. $\quad$ Engel J. Surgery for Seizures. N Engl J Med. 1996;334(10):647-653. doi:10.1056/NEJM199603073341008.

2. Luders HO, Awad IA. Conceptual considerations. In: Luders H, ed. Epilepsy Surgery. New York: Raven Press; 1991:51-62.

3. Rasmussen T. Tailoring of cortical excision for frontal lobe epilepsy. Can J Neurol Sci. 1991;18(4 Suppl):606-610.

4. Laskowitz DT, Sperling MR, French JA, O'Connor MJ. The syndrome of frontal lobe epilepsy: characteristics and surgical management. Neurology. 1995;45(4):780-787. doi:10.1212/WNL.45.4.780.

5. Englot DJ, Wang DD, Rolston JD, Shih TT, Chang EF. Rates and predictors of long-term seizure freedom after frontal lobe epilepsy surgery: a systematic review and meta-analysis. $J$ Neurosurg. 2012;116(5):1042-1048. doi:10.3171/2012.1.JNS111620.

6. Binnie CD, Polkey CE. Surgery for epilepsy. In: Kennard C, ed. Recent Advances in Clinical Neurology. London: Churchill Livingstone; 1992:55-93.

7. Hosking PG. Surgery for frontal lobe epilepsy. Seizure. 2003;12:160-166. doi:10.1016/S10591311(02)00189-9.

8. Talairach J, Bancaud Jean, Bonis A, et al. Surgical therapy for frontal epilepsies. Adv Neurol. 1992;57:707-732.

9. Aubert S, Wendling F, Regis J, et al. Local and remote epileptogenicity in focal cortical dysplasias and neurodevelopmental tumours. Brain. 2009;132(11):3072-3086. doi:10.1093/brain/awp242.

10. Bartolomei F, Chauvel P, Wendling F. Epileptogenicity of brain structures in human temporal lobe epilepsy: a quantified study from intracerebral EEG. doi:10.1093/brain/awn111.

11. Engel Jr J, Van Ness PC, Rasmussen TB OL. Outcome with respect to epileptic seizure. In: Engel J, ed. Surgical Treatment of the Epilepsies. Raven Pres. New York; 1993:602-622.

12. Husson F, Lê S, Pagès J. Exploratory Multivariate Analysis by Example Using R. Chapman \&. (Blei D, Madigan D, eds.). London: Chapman \& Hall/CRC; 2010.

13. Carvalho H. Multivariate Analysis of Qualitative Data. (Sílabo E, ed.). Lisbon; 2004.

14. Andersen PK, Gill RD. Cox's regression model for counting processes: a large sample study. Ann Stat. 1982;10(4):1100-1120.

15. Hothorn T, Hornik K, Zeileis A. Unbiased Recursive Partitioning: A Conditional Inference Framework. J Comput Graph Stat. 2006;15(3):651-674. doi:10.1198/106186006X133933.

16. Sadek AR, Gray WP. Chopping and changing: Long-term results of epilepsy surgery. Lancet. 2011;378(9800):1360-1362. doi:10.1016/S0140-6736(11)61593-6.

17. De Tisi J, Bell GS, Peacock JL, et al. The long-term outcome of adult epilepsy surgery, patterns of seizure remission, and relapse: A cohort study. Lancet. 2011;378(9800):1388-1395. doi:10.1016/S0140-6736(11)60890-8.

18. McIntosh AM, Kalnins RM, Mitchell LA, Fabinyi GCA, Briellmann RS, Berkovic SF. Temporal lobectomy: Long-term seizure outcome, late recurrence and risks for seizure recurrence. Brain. 2004;127(9):2018-2030. doi:10.1093/brain/awh221.

19. McIntosh AM, Averill CA, Kalnins RM, et al. Long-term seizure outcome and risk factors for recurrence after extratemporal epilepsy surgery. Epilepsia. 2012;53(6):970-978. doi:10.1111/j.1528-1167.2012.03430.x.

20. Jeha LE, Najm I, Bingaman W, Dinner D, Widdess-Walsh P, L??ders H. Surgical outcome and prognostic factors of frontal lobe epilepsy surgery. Brain. 2007;130(2):574-584. doi:10.1093/brain/awl364.

21. Tassi L, Colombo N, Garbelli R, et al. Focal cortical dysplasia: neuropathological subtypes, EEG, neuroimaging and surgical outcome. Brain. 2002;125(8):1719-1732.

22. Awad IA, Rosenfeld J, Ahl J, Hahn JF, Lüders H. Intractable Epilepsy and Structural Lesions of the Brain: Mapping, Resection Strategies, and Seizure Outcome. Epilepsia. 1991;32(2):179186. doi:10.1111/j.1528-1157.1991.tb05242.x.

23. Janszky J, Jokeit H, Schulz R, Hoppe M, Ebner A. EEG predicts surgical outcome in lesional frontal lobe epilepsy. Neurology. 2000;54(7):1470-1476. doi:10.1212/WNL.54.7.1470. 
24. Lee JJ, Lee SK, Lee S-Y, et al. Frontal lobe epilepsy: clinical characteristics, surgical outcomes and diagnostic modalities. Seizure. 2008;17(6):514-523. doi:10.1016/j.seizure.2008.01.007.

25. Mosewich RK, So EL, O'Brien TJ, et al. Factors predictive of the outcome of frontal lobe epilepsy surgery. Epilepsia. 2000;41(7):843-849.

26. Ferrier CH, Engelsman J, Alarcón G, Binnie CD, Polkey CE. Prognostic factors in presurgical assessment of frontal lobe epilepsy. J Neurol Neurosurg Psychiatry. 1999;66(3):350-356. doi:10.1136/jnnp.66.3.350.

27. Kim CH, Chung CK, Lee SK. Longitudinal change in outcome of frontal lobe epilepsy surgery. Neurosurgery. 2010;67(5):1222-1229. doi:10.1227/NEU.0b013e3181f2380b.

28. Yun CH, Lee SK, Lee SY, Kim KK, Jeong SW, Chung CK. Prognostic factors in neocortical epilepsy surgery: Multivariate analysis. Epilepsia. 2006;47(3):574-579. doi:10.1111/j.15281167.2006.00470.x.

29. McGonigal A, Bartolomei F, Régis J, et al. Stereoelectroencephalography in presurgical assessment of MRI-negative epilepsy. Brain. 2007;130(12):3169-3183. doi:10.1093/brain/awm218.

30. Lazow SP, Thadani VM, Gilbert KL, et al. Outcome of frontal lobe epilepsy surgery. Epilepsia. 2012;53(10):1746-1755. doi:10.1111/j.1528-1167.2012.03582.x.

31. Simasathien T, Vadera S, Najm I, Gupta A, Bingaman W, Jehi L. Improved outcomes with earlier surgery for intractable frontal lobe epilepsy. Ann Neurol. 2013;73(5):646-654. doi:10.1002/ana.23862.

32. Schramm J, Kral T, Kurthen M, Blümcke I. S Urgery To T Reat F Ocal F Rontal L Obe E Pilepsy. 2002;51(3):644-655. doi:10.1227/01.NEU.0000024154.71205.EE.

33. Zaatreh MM, Spencer DD, Thompson JL, et al. Frontal lobe tumoral epilepsy: Clinical, neurophysiologic features and predictors of surgical outcome. Epilepsia. 2002;43(7):727-733. doi:10.1046/j.1528-1157.2002.39501.x.

34. Elsharkawy AE, Alabbasi AH, Pannek H, et al. Outcome of frontal lobe epilepsy surgery in adults. Epilepsy Res. 2008;81(2-3):97-106. doi:10.1016/j.eplepsyres.2008.04.017.

35. Holtkamp M, Sharan A, Sperling MR. Intracranial EEG in predicting surgical outcome in frontal lobe epilepsy. Epilepsia. 2012;53(10):1739-1745. doi:10.1111/j.15281167.2012.03600.x.

36. Alomar S, Jones J, Maldonado A, Gonzalez-Martinez J. The Stereo-Electroencephalography Methodology. Neurosurg Clin N Am. 2016;27(1):83-95. doi:10.1016/j.nec.2015.08.003.

37. Jayakar P, Gotman J, Harvey AS, et al. Diagnostic utility of invasive EEG for epilepsy surgery: Indications, modalities, and techniques. Epilepsia. 2016:1-13. doi:10.1111/epi.13515.

38. Podkorytova I, Hoes K, Lega B. Stereo-Encephalography Versus Subdural Electrodes for Seizure Localization. Neurosurg Clin N Am. 2016;27(1):97-109. doi:10.1016/j.nec.2015.08.008.

39. Wilder B, King R, Schmidt R. Cortical and subcortical secondary epileptogenesis. Neurology. 1969;19:643-652.

40. Tassi L, Pasquier B, Minotti L, et al. Cortical dysplasia: Electroclinical, imaging, and neuropathologic study of 13 patients. Epilepsia. 2001;42(9):1112-1123. doi:10.1046/j.15281157.2001.00501.x.

41. Colombo N, Tassi L, Galli C, et al. Focal Cortical Dysplasias: MR Imaging, Histopathologic, and Clinical Correlations in Surgically Treated Patients with Epilepsy. AJNR Am J Neuroradiol. 2003;24:724-733.

42. Alarcón G, Valentín A, Watt C, et al. Is it worth pursuing surgery for epilepsy in patients with normal neuroimaging? J Neurol Neurosurg Psychiatry. 2006;77:474-480. doi:10.1136/jnnp.2005.077289.

43. Nobili L, Francione S, Mai R, et al. Surgical treatment of drug-resistant nocturnal frontal lobe epilepsy. Brain. 2007;130(2):561-573. doi:10.1093/brain/awl322.

44. Bulacio JC, Jehi L, Wong C, et al. Long-term seizure outcome after resective surgery in patients evaluated with intracranial electrodes. Epilepsia. 2012;53(10):1722-1730. doi:10.1111/j.1528-1167.2012.03633.x.

45. Bonini F, McGonigal A, Wendling F, et al. Epileptogenic networks in seizures arising from motor systems. Epilepsy Res. 2013;106(1-2):92-102. 
2

3 Figure 1. Kaplan-Meier "survival" probability estimate (red line) with 95\% confidence bounds (dotted

4 lines) in the overall population, where event is transition to an Engel class greater than one. Censored

5 data are marked by crosses. Median survival time estimate $=3$ years.

6

7 Figure 2. Factorial plan of association of clinical variables with seizure outcome. The graphic is

\section{Figure legends} created by the two dimensions derived from the multiple correspondence analysis. The horizontal axis represents the first dimension, while the vertical axis represents the second dimension

Figure 3. Kaplan-Meier survival curve showing chances of post-operative seizure freedom depending on the completeness of the resection (Log-rank test: $\chi^{2}=18.5,1 \mathrm{df}, \mathrm{p}<0.001$ ). Median time of recurrence for incomplete resection is 6 months (95\% CI 0.5-2). With complete resection, $67 \%$ of patients are seizure free 10 years after surgery.

Figure 4. Conditional inference tree predicting probability of seizure recurrence: the 'tree' graph shows the hierarchy of significant variables associated with outcome 